\title{
A Network Lifetime-Oriented Energy-Aware Sink Relocation Scheme
}

\author{
http://dx.doi.org/10.3991/ijoe.v11i9.5062 \\ Lin Qiu ${ }^{1}$, Jiangfeng Qin*², Ronghui $\mathrm{Li}^{2}$ \\ ${ }^{1}$ Guangxi Agricultural Vocational College, Nanning, Guangxi, China \\ ${ }^{2}$ Guangxi Institute of Water Resources Research, Nanning, Guangxi, China
}

\begin{abstract}
When sensor nodes are located around a sink node in a wireless sensor network (hereafter referred to as WSN), their energy will be consumed more quickly. Hence it's necessary to make a proper relocation of the sink node to prevent the surrounding sensor nodes from excessive energy consumption. In view of this, this paper proposes an energy-aware sink relocation (hereafter referred to as EASR) scheme, which is able to realize the adaptive adjustment on the transmission range according to the energy status of the sensor nodes in addition to the establishment of conditions for sink relocation. If the relocation condition is met, sink node will move toward the optimal direction based on the energy status of the surrounding sensor nodes. The simulation result shows that the EASR scheme proposed in this paper is able to prolong effectively the network lifetime.
\end{abstract}

Index Terms-Energy-aware routing, Network lifetime, Sink node, Wireless sensor network.

\section{INTRODUCTION}

There are various hardware platforms of wireless sensor network (WSN) nodes now at home and abroad, represented by Mica, Telos, IRIS and Imote2. The major difference between those platforms lies in the different processors and wireless communication modules adopted. Nodes such as Imote2 are not applicable to the energyconstrained environment due to its high performance at the cost of large power consumption, while nodes such as Telos and Mica have lagged behind the existing design level of integrated circuit industry for being designed long ago. The WSN nodes are usually powered by battery, and operate under hostile environment in large amounts. In addition, it is quite difficult to replace such WSN nodes. Based on the reasons above, low power consumption is the most important design criteria for wireless sensor networks.

WSNs consist of numerous wireless sensor nodes that are small in size with finite energy and able to provide wireless communication. In a WSN, all of these nodes are working to detect the abnormal events such as forest fire or collect data [1] from the environment such as temperature and humidity, and then send the information (the abnormal events or the environmental data) to the special nodes that are also called as the sink nodes for the transmission of information to the supervisor through the internet. As shown in Figure 1, when an abnormal event is detected, the node $e$ will send the information through a multi-hop path $(e \rightarrow d \rightarrow c \rightarrow b \rightarrow a$ ) to the sink node, which will then transmit the information to the Supervisor through the internet.

Generally, sensor nodes are deployed in a field environment, where it would be very hard for them to be reenergized as soon as they run out of energy. When energy depletion occurs, sensor nodes will become unable to transmit or collect any information, which would give rise to numerous problems such as Coverage Holt that might lead to the failure in communication [2-3]. On account of this, now lots of researchers are devoting themselves to the research on how to preserve energy in the sensor nodes, or in other words, how to utilize node energy efficiently in a reasonable way. For example, design a duty cycle scheduling so that nodes can periodically enter into the sleeping mode to save the node energy. Of course, during the conservation of node energy, nodes in the WSNs won't be affected and they will continue to work normally [4-5]. In Literature [6-9], it adopts the energy-efficient routing algorithm in the design to balance the energy consumption in every sensor node.

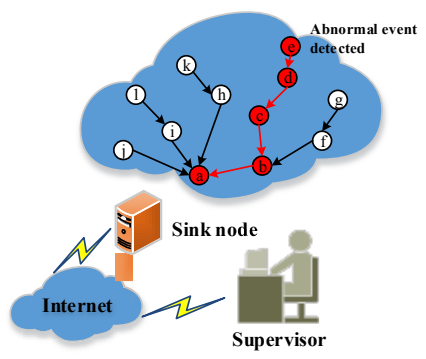

Figure 1. Operation sketch map of sink node in a WSN

Moreover, it's applicable to relocate the sensor nodes to save node energy [10]. Although such a scheme is able to prolong the lifetime of the WSNs, it might lead to additional energy consumption by the sensor nodes. In view of this, Literature [11-15] proposes a compromise scheme: Relocate sink node instead of any sensor node as shown in Figure 2.

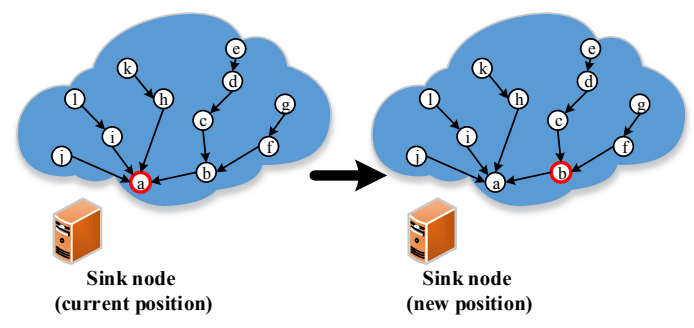

Figure 2. Sketch map of sink relocation 
As shown in Figure 2, after information has been transferred several times, Node a, which is a node nearest to the sink node, runs out of energy. Upon energy depletion, the sink node fails to receive any new information, bringing the whole MSN to a halt. In this case, call the region where Node a is located as the hotspot. Before Node a should run out of energy in the hotspot, it's applicable to relocate the sink node to reduce the burden on Node a. As shown in Figure 2, relocate the sink node to a place near to Node $b$. Then Node $b$ will assume the task to transmit the information to the sink node. In this way, the energy of Node a will be conserved and the working life of the whole WSN will be extended.

In view of this, this paper proposes a new sink relocation scheme that is also called as the EASR scheme, which is able to provide guidance on when and where the sink node can be relocated. According to this scheme, the sink node will move to reduce the burden on the neighboring sensor nodes and preserve the energy in them according to the information that has been detected about the energy in the neighboring sensor nodes. Meanwhile, it will make an adjustment on the transmission range of the sensor nodes based on the energy level in them. The numerical simulation reveals that the EASR scheme proposed in this paper is able to extend effectively the working life of the whole WSNs.

\section{EASR}

In our EASR scheme, first adjust the transmission range of a sensor node according to its residual battery energy (hereafter referred to as RBE), or in other words, adaptively change the transmission range. Battery energy becomes less after the node has transmitted the information several times or after it has completed the detection task. Then in order to conserve the energy, it's necessary to reduce the transmission range. After that, relocate the sink node and implement the MCP (Maximum Capacity Path) routing protocol [6] to spread the message and thus extend the lifetime of the network. Take note that the message transmission routing algorithm might affect the performance of the whole system (sink relocation or message forwarding). Yet despite all this, our EASR scheme can still be integrated with multiple existing routing algorithms. However in this paper, only the MCP routing protocol is adopted to reduce the influence on the system performance since the parameters applied in the MCP routing protocol agree with the decisive parameters used in the EASR scheme proposed in this paper.

Our EASR scheme consists of two parts: the adaptive change of transmission range and sink relocation.

\section{A. Adaptive change of transmission range}

Generally, with the increase in the transmission range of the sensor node, more neighbor nodes will be observed, which will also require an improvement in the quality of the router. However the long transmission range also brings about some deficiencies, especially that it will lead to a long message transmission range at every hop with more battery energy to be consumed. But in the opposite situation, if the transmission range is rather short, it will facilitate the conservation of the battery energy although it's not helpful to the router.
Hence, as shown in Figure 3, the sensor nodes will be classified into three groups according to the energy capacity of battery in the sensor nodes in an EASR scheme.

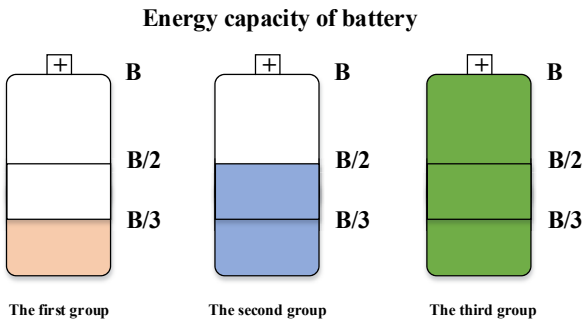

Figure 3. Classification of sensor nodes in an EASR scheme

Assume that $B$ represents the gross energy capacity of battery and $r(u)$ represents the current residual battery capacity of the sensor nodes. Then the sensor node $i$ can be grouped into $\mathfrak{\Im}^{L}, \mathfrak{\Im}^{M}$ and $\mathfrak{\Im}^{H}$ according to the value of $r(u)$ :

(1) In the case that $0 \leq r(u) \leq B / 3$, the sensor nodes will be grouped into $\Im^{L}$, say $i \in \mathfrak{\Im}^{L}$.

(2) In the case that $B / 3 \leq r(u) \leq B / 2$, the sensor nodes will be grouped into $\mathfrak{I}^{M}$, say $i \in \mathfrak{S}^{M}$.

(3) In the case that $B / 2 \leq r(u) \leq B$, the sensor nodes will be grouped into $\mathfrak{S}^{H}$, say $i \in \mathfrak{S}^{H}$.

Grouped into $\mathfrak{I}^{L}, \mathfrak{I}^{M}$ and $\mathfrak{\Im}^{H}$, the sensor nodes have different transmission ranges. Assume that the maximum transmission range of a sensor node is $\gamma$. Then the transmission ranges of the sensor nodes that are respectively grouped into $\mathfrak{\Im}^{L}, \mathfrak{\Im}^{M}$ and $\mathfrak{\Im}^{H}$ are separately $\gamma / 4, \gamma / 2$ and $\gamma$. Table I shows the pseudo-code of the algorithm for the adaptive change of the transmission range.

TABLE I. ALGORITHM FOR THE ADAPTIVE CHANGE OF TRANSMISSION RANGE

Input: $\gamma$ the initial transmission range, $B$ the initial battery energy and $r(u)$ the current battery energy,

Output: $R$ the transmission range

$$
\begin{aligned}
& \text { If } 0 \leq r(u) \leq B / 3 \text { then } \\
& \begin{array}{l}
R=\gamma / 4 \\
\text { elseif } B / 3 \leq r(u) \leq B / 2 \text { then } \\
R=\gamma / 2 \\
\text { elseif } B / 2 \leq r(u) \leq B \text { then } \\
R=\gamma \\
\text { End }
\end{array}
\end{aligned}
$$

Analysis as above shows that in the beginning when the sensor nodes are battery powered with sufficient energy, it's feasible to adopt the maximum transmission range $\gamma$ to shorten the routing path. However when the battery power drops, the transmission range will become shortened to 
save the energy. Therefore such an adaptive change of transmission range is able to prolong efficiently the network lifetime.

\subsection{Sink relocation algorithm}

Sink relocation algorithm is involved with two aspects. First, it decides if sink relocation can be triggered. That's to say, it will detect if the conditions for sink relocation have been met. After that, it will determine the moving direction and distance of a sink node.

\section{(1) Conditions for sink relocation}

Sink node is able to collect periodically the residual battery energy $r(u)$ of every sensor node in the network. After data collection, the sink node will calculate $P_{u s}^{*}$, the maximum capacity path according to the MCP routing protocol, where $S$ represents the sink node and $u$ is the neighbor node of the sink. With regard to the maximum capacity path $P_{u s}^{*}$, define a maximum capacity value $c\left(P_{u s}^{*}\right)[6]$ to it, and assume that information about the battery capacity of $N$ sensor nodes has been collected. Sink relocation will be triggered if one of the following two conditions can be met. 1) When $c\left(P_{u s}^{*}\right)$ of a random node among the $N$ sensor nodes is lower than $B / 2$. 2) When $r(u)$, the average residual battery capacity of $N$ sensor nodes is below $B / 2$. Both of the conditions can be formally represented by:

$$
\begin{aligned}
& \exists u \in N, c\left(P_{u s}^{*}\right)<B / 2 \\
& \sum_{u \in N} r(u) /|N|<B / 2
\end{aligned}
$$

When either of the above conditions is met, it means that the residual battery capacity of the neighbor nodes around the sink is at a low level or the residual battery capacity of some paths is below $B / 2$. Under such a circumstance, it's necessary to relocate the sink node to a proper place so as to prolong the network lifetime.

(2) Moving distance and direction of a sink node

Before a sink node is relocated, it's necessary to decide the moving distance and direction of the sink node. Define that distance of every movement by the sink node is $r$ in four directions, including rightward, leftward, upward and downward. As shown in Figure 4, assume that $S_{C 1}, S_{C 2}, S_{C 3}$ and $S_{C 4}$ are separately the moving destinations of the sink node after the above four moving directions and distances are defined. At first, when the sink node is located at the center, it can be moved in the following four directions, which separately are rightward, leftward, upward and downward at a moving distance of $r$ under the condition that $r=\gamma$.

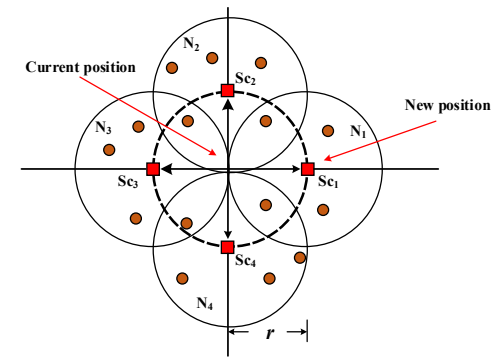

Figure 4. Moving distances and directions of the sink node
Define the neighboring subset $N_{k}$ and when $1 \leq k \leq 4$, it indicates the number of the neighbors centered by the sink node with a radius of $r$. Correspondingly, define the weight coefficient as $\omega_{k}$ :

$$
\omega_{k}=\min \left\{c\left(P_{u s}^{*}\right) \mid u \in N_{k}\right\}
$$

Assume that the moving destination of a sink node is $S_{C k^{*}}$, which is just in one of the following four directions, say $S_{C 1}, S_{C 2}, S_{C 3}$ and $S_{C 4}$. Actually in what direction a sink node should move is subject to four weight coefficients $\omega_{k}(1 \leq k \leq 4)$. Therefore the moving direction $D r$ is:

$$
D r=\left\{k \mid \max _{1 \leq k \leq 4}\left\{\omega_{k}\right\}\right\}
$$

As shown in Formula (4), the sink node moves along the direction where the weight coefficient $\omega_{k}$ reaches the maximum. Table II shows the pseudo-code of the sink relocation algorithm.

TABLE II. THE PSEUDO-CODE OF THE SINK RELOCATION ALGORITHM

Sink node relocation algorithm

Input: $\gamma$ the initial transmission range, $B$ the initial battery energy, $r(u)$ the current battery energy,

$V$ the set of the sensor nodes in WSNs

Output: $\mathrm{Dr}$ the moving direction

Collecting the residual battery energy $r(u), \forall u \in V$

Then, compute $c\left(P_{u s}^{*}\right), \forall u \in V$

If $\exists u \in N, c\left(P_{u s}^{*}\right)<B / 2$ or $\sum_{u \in N} r(u) /|N|<B / 2$ is true

Then ,compute $\omega_{k}=\min \left\{c\left(P_{u s}^{*}\right) \mid u \in N_{k}\right\}$

and ,compute $D r=\left\{k \mid \max \left\{\omega_{k}\right\}\right\}$

\section{NUMERICAL ANALYSIS}

In order to make an intensive analysis on the performance of the EASR scheme, three scenarios have been set up in this paper for the simulation in addition to the comparison of this EASR scheme with the one-step moving scheme [15] and the stationary sink scheme, where the sink node remains static all the time without any movement. All of these three schemes adopt MCP routing protocol to transmit message. In addition, all of the sensor nodes are motionless after the deployment in these three schemes. However sink nodes can be relocated in the other two schemes except the stationary scheme. In both of the one-step moving scheme and the stationary scheme, $\gamma$ the transmission range of the sensor node and the sink node remains unchanged, while it varies against the status of the battery capacity in the EASR scheme. As described below, this paper makes an analysis on the performance and the lifetime of the network based on three different scenarios.

\section{(1) Scenarios 1}

Scenarios 1 gives all of the parameters used, where the initial battery energy of the node $B=1000 \mathrm{~J}$, the transmission range $\gamma=25$ meters and the number of the 
sensor nodes varies from 50, 75, 100, 125 to 150 . Simulation is conducted in an area of $100 * 100$ with the result shown in Figure 5.

Figure 5 shows that the EASR scheme proposed in this paper outperforms the one-step moving scheme and the stationary scheme when sensor nodes are within the entire range of variation. As expected, the stationary scheme shows the poorest performance since in a stationary scheme, the sink node is located in a fixed position, bringing about fixed neighbor nodes (hot-spots) that consume battery energy very quickly due to the continuous message forwarding by them.

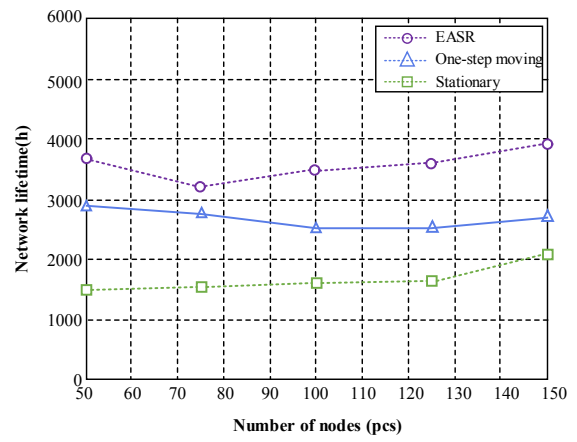

Figure 5. Changes in the network lifetime against the number of the sensor nodes

(2) Scenarios 2

Table III gives all of the parameters used in Scenarios 2. Different from Scenarios 1, Scenarios 2 reveals that the initial battery energy is variable in the study of the impact of the battery energy on the network lifetime with the simulation result shown in Figure 6.

TABLE III.

PARAMETERS APPLIED IN SCENARIOS 2

\begin{tabular}{|c|c|}
\hline Parameters & Values \\
\hline$(\mathrm{J})$ Initial energy & 500、750、1000、1250、1500 \\
\hline$(\mathrm{m})$ Transmission range & $100 \times 100$ \\
\hline$\left(\mathrm{m}^{2}\right)$ Simulation area & 100 \\
\hline Number of nodes & \\
\hline
\end{tabular}

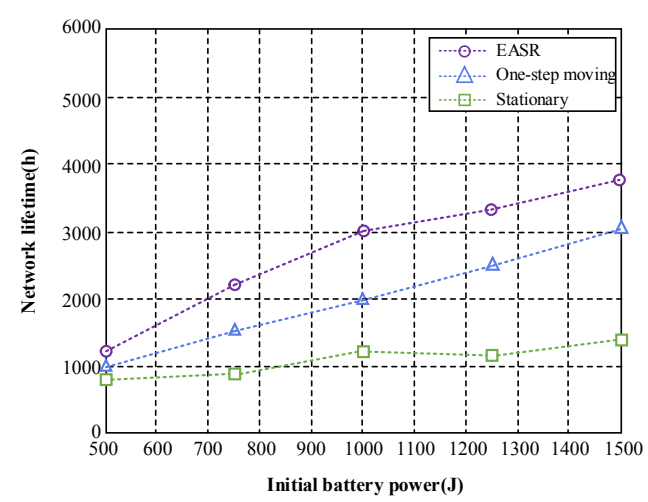

Figure 6. Changes in the network lifetime against the initial battery power

Figure 6 shows that network lifetime can be extended with the increase in the initial battery energy of the sensor nodes. Compared with the one-step moving scheme and the stationary scheme, the EASR scheme proposed in this paper shows the optimal performance, having more obvious advantages in the performance with the increase in the initial battery energy. Similar to Scenarios 1, Scenarios 2 also shows that the stationary scheme has the worst performance.

(2) Scenarios 3

Table IV gives all of the simulation parameters used in Scenarios 3, where the simulation is performed to study the influence of the transmission range of the nodes on the network lifetime with the simulation result shown in Figure 7.

TABLE IV.

PARAMETERS APPLIED IN SCENARIOS 3

\begin{tabular}{|c|c|}
\hline Parameters & Values \\
\hline$(\mathrm{J})$ Initial energy & 1000 \\
\hline$(\mathrm{m})$ Transmission range & $20 、 25 、 30 、 35$ \\
\hline$\left(\mathrm{m}^{2}\right)$ Simulation area & $200 \times 200$ \\
\hline Number of nodes & 400 \\
\hline
\end{tabular}

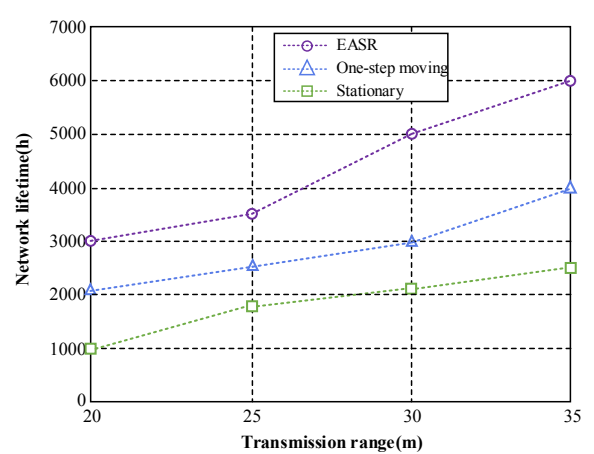

Figure 7. Changes in the network lifetime against the transmission range of the nodes

As shown in Figure 7, network lifetime can be improved with the increase in the transmission range of the nodes. This is because that with the increase in the transmission range, the length of the routing path will be shortened to increase accordingly the number of the neighbor nodes near to a sink, leading to an increase in the residual battery energy of the nodes in the hot spots, where messages are forwarded to the sink node. Moreover, compared with the one-step moving scheme and the stationary scheme, the EASR scheme proposed in this paper is able to prolong the network lifetime.

\section{CONCLUSIONS}

The speed at which the sensor nodes run out of battery energy affects severely the lifetime of a sensor network. Currently most of the researchers focus on their researches on the preservation of battery energy through the energyaware routing method to prolong the network lifetime. However according to the EASR scheme proposed in this paper, sink nodes will move according to the energy status of the neighbor nodes to reduce the energy consumption in them and thus to prolong finally the network lifetime. The simulation result shows that our EASR scheme is able to improve efficiently the network lifetime. Actually in all of the three scenarios, this EASR scheme is able to guarantee long network lifetime. 


\section{REFERENCES}

[1] Kegami T. An overview of the sensor networks [J]. IEEJ Transactions on Electronics, Information and Systems, 2008, 128(10): 1498 -1503. http://dx.doi.org/10.1541/ieejeiss.128.1498

[2] Shen Yanxia, Xue Xiaosong. Path optimization strategy of WSNs mobile beacon nodes[J]. Transducer and Microsystem Technologies, 2012,31(12): 42-46.

[3] Cui Huanqing, Wang Yinglong, Guo Qiang, LV Jialiang. Multimobile-beacon assisted distributed node localization scheme $[\mathrm{J}]$. Journal on Communications, 2012,33(3):103-112.

[4] Zou Y, Chakrabarty K.A distributed coverage and connectivity centric technique for selecting active nodes in wireless sensor networks [J]. IEEE Trans. Comput. 2005, 54(8):978-991. http://dx.doi.org/10.1109/TC.2005.123

[5] Delicato F, Protti F, Pirmez L.An efficient heuristic for selecting active nodes in wireless sensor networks [J]. Comput. Netw.2006, 50(18):

3701-3720. http://dx.doi.org/10.1016/j.comnet.2006.04.005

[6] Huang S C, Jan R H. Energy-aware, load balanced routing schemes for sensor networks [C]. in Proc. 10th Int. Conf. Parallel Distrib. Syst., Jul. 2004: 419-425.

[7] Karkvandi H R, Pecht E, Pecht O Y. Effective lifetime-aware routing in wireless sensor networks [J]. IEEE Sensors J., 2011, 11(12): 3359-3367. http://dx.doi.org/10.1109/JSEN.2011.2159110

[8] AlShawi I S, Lianshan Y, Wei P, Bin L. Lifetime enhancement in wireless sensor networks using fuzzy approach and A-star algorithm [J]. IEEE Sensors J.2012, 12(10): 3010-3018. http://dx.doi.org/10.1109/JSEN.2012.2207950

[9] Wang S S, Chen Z P.LCM: A link-aware clustering mechanism for energy-efficient routing in wireless sensor networks [J]. IEEE $\begin{array}{llll}\text { Sensors J. } & \text { 2013, } 2 \text { 13(2): }\end{array}$ http://dx.doi.org/10.1109/JSEN.2012.2225423

[10] Mousavi H, Nayyeri A, Yazani N, Lucas C.Energy conserving movement-assisted deployment of ad hoc sensor networks [J]. IEEE Commun. Lett., 2006, 10(4): 269-271. http://dx.doi.org/10.1109/LCOMM.2006.1613743

[11] Wang G L, Cao G H, Porta T L, Zhang W S. Sensor relocation in mobile sensor networks [J]. in Proc. IEEE Inf. Commun. Conf., 2005, (4): 2302-2312.
[12] Somasundara A A, Kansal A, Jea D D, Estrin D, Srivastavam M B. Controllably mobile infrastructure for low energy embedded networks [J]. IEEE Trans. Mobile Comput., 2006, 5(8): 958-973. http://dx.doi.org/10.1109/TMC.2006.109

[13] Yang Y, Fonoage M I, Cardei M. Improving network lifetime with mobile wireless sensor networks [J]. Comput. Commun., 2010, 33(4): 409-419. http://dx.doi.org/10.1016/j.comcom.2009.11.010

[14] Ding J W, Deng D J, Wu T Y, Chen H H.Quality-aware bandwidth allocation for scalable on-demand streaming in wireless networks [J]. IEEE J. Sel. Areas Commun., 2010, 28(3): 366-376. http://dx.doi.org/10.1109/JSAC.2010.100408

[15] Sun Y, Huang W F, Sun L. Moving schemes for mobile sinks in wireless sensor networks [J]. in Proc. IEEE IPCCC, 2007: 101108.

\section{AUTHORS}

Lin Qiu, female, is with Guangxi Agricultural Vocational College, Nanning, Guangxi, 530007, China. She was born in Guangxi Province of China, is a lecturer with master' degree, absorbed in computer networks and E-commerce research. (346448337@qq.com)

Jiangfeng Qin, male, is with Guangxi Institute of Water Resources Research, Nanning, Guangxi, 530023, China. He was born in Guangxi Province of China, is an engineer with master' degree, absorbed in computer networks and automatic control technologies research. (8187282@qq.com)

Ronghui Li, male, is with Guangxi Institute of Water Resources Research, Nanning, Guangxi, 530023, China. $\mathrm{He}$ was born in Guangxi Province of China, is a senior engineer with doctor' degree, absorbed in hydraulic and ecological modeling research. (yahui28@gmail.com)

The authors acknowledge the support of the National Natural Science Foundation of China (NSFC) NO.51409050 \& NO.51569006. Submitted 21 September 2015. Published as resubmitted by the authors 20 October 2015 . 